\title{
La prevención del abuso de drogas en Castilla y León: Análisis descriptivo de los programas desarrollados entre 1986 y 1998
}

\author{
Martínez González, F. \\ Jefe de Servicio de Prevención, Asistencia e Integración de Drogodependientes. Consejería de Sanidad y Bienestar Social de la Junta de Castilla y León. \\ Enviar correspondencia a: \\ Fernando Martínez González. Jefe del Servicio de Prevención, Asistencia e Integración de Drogodependientes. Consejería de Sanidad y Bienestar \\ Social de la Junta de Castilla y León. Francisco Suárez, nº 2 - derecha. 47071 - Valladolid.
}

\section{RESUMEN:}

En el presente estudio se hace un análisis descriptivo de 143 programas de prevención del uso indebido de drogas y de sus problemas asociados desarrollados en Castilla y León con financiación de la Administración Autonómica en el período comprendido entre 1986 y 1998. Junto a hechos positivos como un incremento muy notable de la actividad preventiva basada en múltiples estrategias y de la que se ocupan un número creciente e importante de entidades públicas y privadas, también hay numerosos puntos débiles que es preciso corregir en los próximos años. Entre las principales deficiencias se encuentran la falta de fundamentación teórica de los programas, una definición poco precisa de los objetivos y de la población diana, acciones preventivas estereotipadas y escasamente validadas e importantes carencias en el terreno de la evaluación. Se concluye que las insuficiencias de los programas de prevención en Castilla y León son equiparables a las detectadas en estudios de ámbito nacional y que es preciso dar un salto cualitativo en la prevención de modo que los programas que se desarrollen en el futuro se basen en la evidencia científica.

Palabras clave: Prevención. Castilla y León. Programas. Puntos fuertes y débiles.

\section{ABSTRACT:}

This study presents a descriptive analysis of 143 programs for drug abuse prevention and related problems developed in the region of Castilla and Leon during the period 1986 to 1998 funded by the Autonomous Government. There are positive aspects as a notable increase of the preventing activity based in multiple strategies carried out by an important amount of public and private institutions. On the other hand, there are several weak aspects which it is necessary to correct in the future. Among the main deficiencies there are the lack of theoretical basis of the programs, a poor definition of the aims and the population target, stereotypied preventive actions of unknown value and, finally, important lacks in the ground of the evaluation. The lacks in the programs of Castilla and Leon are similar to those detected in other national estudies; therefore, the prevention efforts may be improved to develop in the future programs based in scientific evidence.

Key words: Prevention. Castilla and Leon. Programs. Strengths and weaknesses.

\section{INTRODUCCIÓN}

D esde la aprobación del Plan Nacional sobre Drogas en julio de 1.985 el número de programas de prevención del uso indebido de drogas ha aumentado considerablemente en España (DGPNSD, 1997). Sin embargo, algunos estudios ponen de manifiesto que la prevención en nuestro país adolece de una serie de defectos que afectan sobre todo a la planificación y a la evaluación de las intervenciones.

Las deficiencias más frecuentemente identificadas tienen que ver con la falta de fundamentación teórica de los programas (Froján y Santacreu, 1.994; March Cerdá y Orte, 1.994); con la ausencia de un diagnosti- co previo de la realidad sobre la que se pretende intervenir (March Cerdá y Orte, 1.994; GID, 1997); con una inadecuada o imprecisa definición de objetivos (GID, 1997) y con severas deficiencias en la evaluación de las intervenciones, en especial en lo referente a los diseños de investigación, al control de la ejecución y a la evaluación de los resultados (Froján y Santacreu, 1.994; March Cerdá y Orte, 1.994; y GID, 1997)

Ante esta situación deficitaria el Plan Nacional sobre Drogas aprobó en diciembre de 1996 un conjunto de criterios básicos de prevención (Ministerio del Interior, 1997) a los que consideró como un primer paso en la tarea de ir mejorando paulatinamente la calidad de los programas preventivos en España. 
En Castilla y León también se ha producido un considerable incremento en la actividad preventiva. Dos circunstancias parecen haber resultado decisivas en este aumento. Por un lado, la entrada en vigor de la Ley 3/1994, de prevención, asistencia e integración social de drogodependientes, en la que las políticas y actuaciones preventivas son consideradas prioritarias y en la que se clarifica el nivel competencial de la Adminsitración Local a la que se reserva un importante papel en prevención (Junta de Castilla y León, 1995). Y, por otro lado, la aplicación de tres Planes autonómicos sobre drogas, el último de los cuales, aprobado en octubre de 1997, define treinta criterios de prevención e incluye ocho programas básicos en el área de la prevención del abuso de drogas y de sus problemas asociados (Junta de Castilla y León, 1998).

Llegados a este punto cabe preguntarse si la calidad y eficacia de los programas en Castilla y León ha mejorado paralelamente al crecimiento de la actividad, y si en esta Comunidad Autónoma se aprecian diferencias significativas en materia de prevención en comparación con los datos disponibles a nivel nacional.

\section{MÉTODO}

Tomando como base el censo de programas de prevención del uso indebido de drogas y de sus problemas asociados desarrollados o financiados por la Consejería de Sanidad y Bienestar Social de la Junta de Castilla y León en el período comprendido entre 1.986 y 1.998 , se ha realizado un análisis descriptivo de una tercera parte de los mismos, siguiendo una metodología similar a la empleada en otros estudios descriptivos realizados a nivel nacional (Zacagnini, Colom y Santacreu, 1.993; Froján y Santacreu, 1.994; March Cerdá y Orte, 1.994; y GID, 1997).

Hay que advertir que en los primeros años objeto de estudio algunas de las actuaciones censadas no podrían considerarse en rigor como programas de prevención. Se trata en muchos casos de actividades puntuales que no alcanzan unos mínimos requisitos metodológicos. Sin embargo, se ha considerado de interés incluirlas al tratarse del reflejo de una época y de una forma de hacer prevención. Para determinar si un programa es o no preventivo se han tenido en cuenta sus objetivos; considerando como prevención toda actuación que persiga evitar el consumo de drogas, retrasar la edad de inicio, evitar la progresión hacia formas problemáticas de uso o reducir los problemas asociados al abuso de drogas.

El objetivo del estudio es describir y analizar los programas de prevención realizados en Castilla y León a lo largo de 13 años, conocer sus características, cómo han ido evolucionando con el paso del tiempo y, a partir de una visión de conjunto, identificar, en el contexto de la prevención en España, las fortalezas y debilidades de las intervenciones preventivas desarrolladas en la Comunidad Autónoma.

El censo a partir del cual se efectúa el análisis no abarca la totalidad de los programas preventivos desarrollados en Castilla y León en el período de tiempo considerado, pero sí analiza la gran mayoría de las intervenciones preventivas llevadas a cabo y todos los programas que se han aplicado dentro del Plan Regional sobre Drogas. Por otra parte, el período de estudio cubre los años en los que en España y en Castilla y León se produce un verdadero vuelco en la intervención en drogodependencias; en esos años se aprueba el primer Plan Nacional sobre Drogas (Ministerio de Sanidad y Consumo, 1985) y los tres Planes Autonómicos hasta el momento desarrollados en Castilla y León (Junta de Castilla y León, 1.989,1.992 y 1998).

Para la selección de programas a analizar se han seguido los siguientes criterios:

a) Incluir todos los programas de carácter regional desarrollados directamente por la Administración Autonómica. Este criterio se ha aplicado por considerar de interés no dejar fuera del análisis ninguno de los programas regionales, dada su cobertura, grado de corresponsabilidad y continuidad en el tiempo, ya que de algún modo suponen un determinado modo de hacer prevención.

b) Elegir al azar de un tercio de los restantes programas, eliminando la misma versión de un programa ya seleccionado y cuya aplicación se prolonga más de un año. Con la aplicación de este criterio se ha pretendido mejorar la representatividad de los programas analizados, evitando que aquellos con más continuidad en el tiempo estuvieran sobrerrepresentados.

Se ha considerado que los programas preventivos desarrollados por una misma entidad a lo largo de varios años tienen distinta naturaleza cuando difieren en sus objetivos, enfoque, población destinataria, actividades o procesos de evaluación. Asimismo, se ha considerado que un mismo programa presenta diferentes versiones cuando, a pesar de tener muchos elementos en común en los aspectos antes enumerados, existen significativas variaciones como resultado del dinamismo y adaptación del programa a una realidad cambiante.

Para la recogida de información de los programas seleccionados se ha utilizado una ficha descriptiva de su contenido diseñada al efecto en la que tienen cabida los aspectos básicos de toda intervención preventiva. Con la utilización de la ficha se ha pretendido sintetizar y homogeneizar al máximo la información 
que se obtenía de los programas, que a veces era muy extensa y variada. La ficha utilizada ha sido necesariamente sencilla, teniendo en cuenta la gran diversidad de programas censados y analizados y que algunos de ellos dejaron de aplicarse hace años, por lo que era probable que no fuera posible conseguir información sobre todas sus características.

La ficha descriptiva registra información sobre variables que se consideran básicas para identificar y caracterizar cualquier programa preventivo.y se basa en sistemas de recogida de información sobre programas de prevención operativos en España (Idea Prevención, 1998). Estas variables son las siguientes:

* Entidad responsable del programa, distinguiendo entre: Administración General del Estado; Administración Autónomica; Diputación Provincial; Ayuntamiento de más de 20.000 habitantes; Ayuntamiento de menos de 20.000 habitantes; ONGs. específicas del campo de las drogodependencias; sindicatos, organizaciones empresariales y profesionales y otras organizaciones de la comunidad.

* Corresponsabilidad, o lo que es lo mismo, si los programas se desarrollan con la participación de varias instituciones o entidades.

* Duración del programa, diferenciando entre programas de menos de un año de duración, programas de uno a tres años de duración y programas de más de tres años de duración.

* Objetivos del programa. El análisis de esta variable se ha limitado a diferenciar si los programas se dirigen a la prevención del abuso de drogas o si tienen por objetivo la reducción de los daños.

* Enfoque o modalidad del programa. Dentro de este aspecto se han considerado tres posibilidades: prevención específica, prevención inespecífica y programas mixtos de prevención específica e inespecífica.

* Estrategia de prevención. Para los programas de prevención primaria se han distinguido seis estrategias: sensibilización, información, educación, alternativas, control y estrategias combinadas.

* Modelo teórico de referencia. En esta variable el análisis se ha centrado únicamente en determinar si en el planteamiento del programa se hace referencia explícita o implícita a un modelo teórico que oriente las actuaciones.

* Drogas cuyos consumos se tratan de prevenir.

* Contexto de intervención, diferenciando entre hábitat (programas urbanos, rurales o rurales y urbanos) y entre ámbitos en los cuales se lleva a cabo el programa, por ejemplo: medios de comunicación, escuela, familia, asociaciones, centros de trabajo, comunidad, etc.
* Población destinataria del programa. En esta variable se ha distinguido entre destinatarios directos o primarios del programa, es decir, la población en la que se quieren lograr los efectos preventivos; y destinatarios indirectos o secundarios, o población intermediaria utilizada para llegar a la población diana o destinatarios primarios.

* Actividades. En la ficha se relacionan 50 tipos de actividades que pueden formar parte de los programas de prevención, dejando, no obstante, una categoría abierta para especificar cualquier otra actividad preventiva distinta.

* Evaluación. Dentro de este apartado la ficha descriptiva indaga sobre los siguientes aspectos: ¿está o no prevista la evaluación?, ¿los objetivos están definidos operativamente?, ¿el programa incluye indicadores de evaluación?, ¿el programa contempla instrumentos y métodos de evaluación?, ¿se especifican las fuentes de información para la evaluación del programa?, ¿se precisa un calendario de evaluación?, y, por último, ¿la evaluación del programa se hace conforme a un diseño de investigación?.

La recogida de información en las fichas descriptivas se ha efectuado a partir de dos tipos de documentos: diseño formal de los programas para solicitar financiación de la Administración Autónomica y memorias en las que se da cuenta de su desarrollo. Hay que advertir que en muchos casos lo que se está analizando es una realidad más teórica que aplicada, indicativa, no obstante, de una forma de entender la prevención y también de la situación de la prevención en un momento dado.

\section{RESULTADOS}

\subsection{Entidades implicadas y programas desarrolla- dos y seleccionados.}

En el período comprendido entre 1986 y 1998 se han censado 434 programas de prevención desarrollados directamente o con financiación de la Consejería de Sanidad y Bienestar Social de la Junta de Castilla y León. Un $64,5 \%$ de estos programas los han desarrollado Administraciones Públicas, entre las que sobresalen los Ayuntamientos, que han asumido el 51,6\% de la actividad preventiva.

Del total de los programas censados han sido seleccionados para su análisis $143(32,9 \%), 8$ de los cuales fueron programas regionales directamente desarrollados por la Administración Autonómica. La distribución por entidades de los programas censados y seleccionados puede verse en la Tabla 1. 
TABLA 1: DISTRIBUCIÓN DE LOS PROGRAMAS PREVENTIVOS SEGÚN LAS ENTIDADES RESPONSABLES (CASTILLA Y LEÓN 1986 - 1998)

\begin{tabular}{||c|cc|cc||}
\hline \multirow{2}{*}{ TIPO DE ENTIDAD RESPONSABLE } & \multicolumn{2}{|c|}{$\begin{array}{c}\text { PROGRAMAS } \\
\text { CENSADOS }\end{array}$} & \multicolumn{2}{c||}{ PROGRACIONAS } \\
\cline { 2 - 5 } & $\mathbf{N}^{\mathbf{0}}$ & $\mathbf{\%}$ & $\mathbf{N}^{\mathbf{0}}$ & $\mathbf{\%}$ \\
\hline Administración Autonómica & 8 & 1,8 & 8 & 5,5 \\
\hline Ayuntamientos $>20.000$ habitantes & 167 & 38,5 & 50 & 35,0 \\
\hline Ayuntamientos $<20.000$ habitantes & 57 & 13,1 & 15 & 10,5 \\
\hline Diputaciones Provinciales & 48 & 11,1 & 14 & 9,8 \\
\hline Total Administraciones Públicas & $\mathbf{2 8 0}$ & $\mathbf{6 4 , 5}$ & $\mathbf{8 7}$ & $\mathbf{6 0 , 8}$ \\
\hline ONGs. especificas del campo de las drogodependencias & 49 & 11,3 & 16 & 11,2 \\
\hline Sindicatos, organizaciones empresariales y profesionales & 8 & 1,8 & 2 & 1,4 \\
\hline Otras organizaciones de la comunidad & 97 & 22,4 & 38 & 26,6 \\
\hline \multicolumn{1}{|c|}{ Total ONGs. } & $\mathbf{1 5 4}$ & $\mathbf{3 5 , 5}$ & $\mathbf{5 6}$ & $\mathbf{3 9 , 2}$ \\
\hline \multicolumn{1}{|c|}{ Totales } & $\mathbf{4 3 4}$ & $\mathbf{1 0 0 , 0}$ & $\mathbf{1 4 3}$ & $\mathbf{1 0 0 , 0}$ \\
\hline
\end{tabular}

En total han sido 118 entidades públicas y privadas de la Comunidad Autónoma las que han desarrollado programas de prevención a lo largo de los 13 años objeto de análisis. Entre las entidades privadas, destacan por su número, las entidades específicas del sector de las drogodependencias (17,8\%), las organizaciones del área de juventud (11\%) y las entidades del campo social y sanitario (8,5\%). En el ámbito de las Administraciones Públicas los Ayuntamientos son los más numerosos, tal y como ocurre al analizar el censo de programas. (Gráfico1).

\section{GRÁFICO 1: ENTIDADES E INSTITUCIONES QUE HAN DESARROLLADO PROGRAMAS DE PREVENCIÓN EN CASTILLA Y LEÓN EN EL PERÍODO COMPRENDIDO ENTRE 1986Y 1998.}

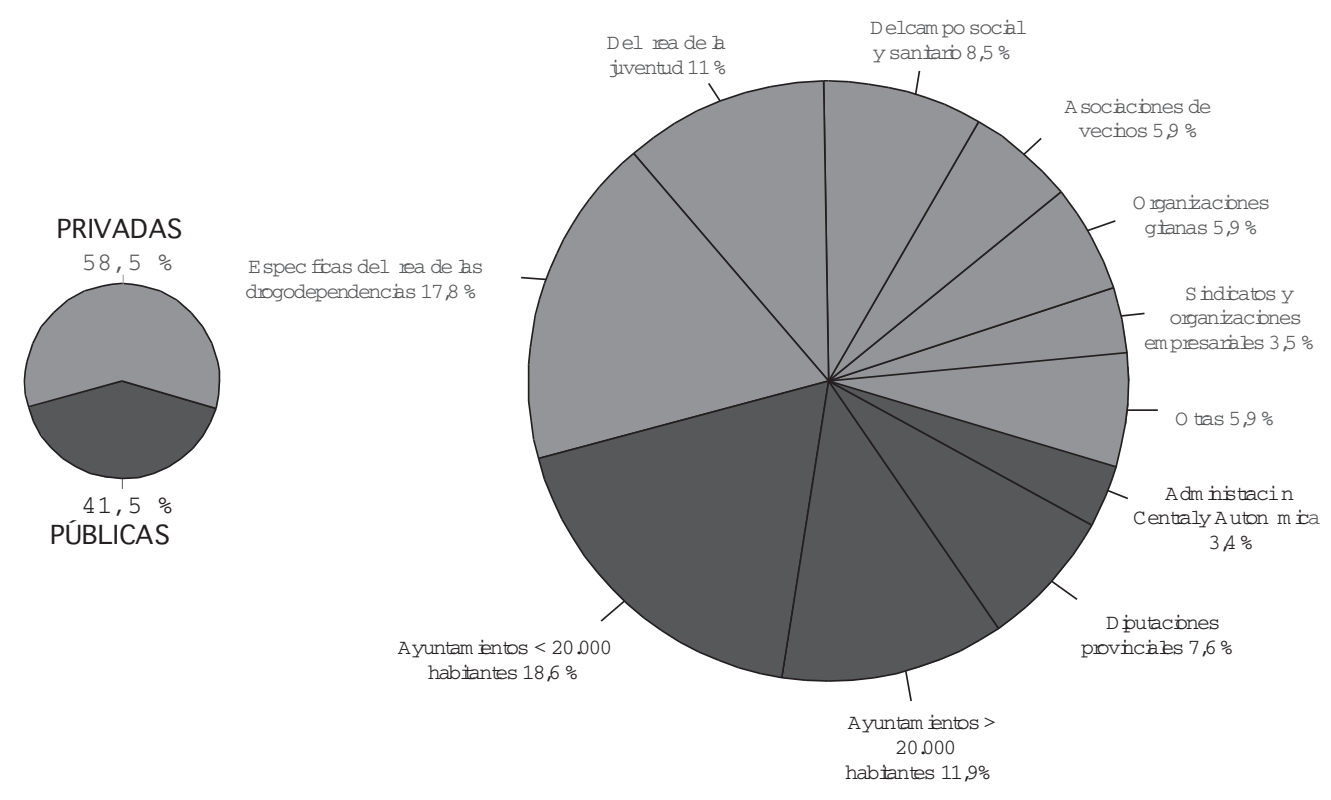


Al considerar la distribución en el tiempo de la actividad preventiva se aprecia como en 1992, coincidiendo con la entrada en vigor del II Plan Regional sobre Drogas, se produce un incremento muy considerable del número de programas desarrollados. Esta notable actividad se mantiene hasta el último año objeto de análisis, si bien es cierto que a partir de 1997 el número de programas decrece por la inclusión de una parte de los mismos en los Planes Municipales y Provinciales sobre drogas (Gráfico 2).

\section{GRÁFICO 2: DISTRIBUCIÓN POR AÑOS DE LOS PROGRAMAS PREVENTIVOS CENSADOSY SELECIONADOS (CASTILLAY LEÓN 1986 - 1998)}

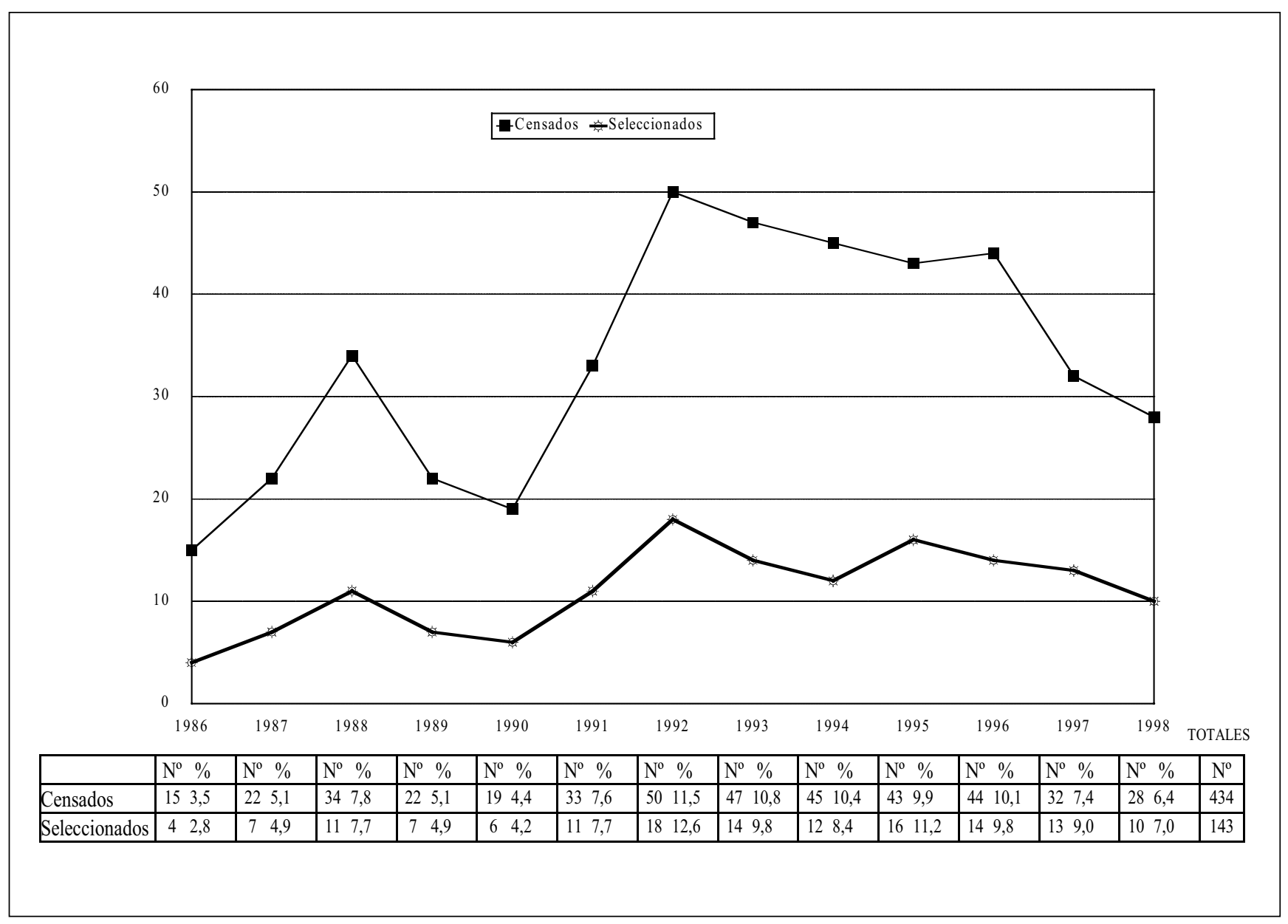

En la mayor parte de los programas ha podido obtenerse información de todas las variables incluidas en la ficha descriptiva. No obstante, en algunas intervenciones preventivas la información no estaba disponible o no ha sido posible inferirla. Las variables más afectadas por falta de datos, con indicación entre paréntesis del porcentaje de programas en los que se carece de información, han sido las siguientes: aspectos relacionados con la evaluación $(13,3 \%)$, drogas cuyos consumos se tratan de prevenir $(11,4 \%)$, corresponsabilidad en la ejecución del programa
$(10,5 \%)$ y ámbitos o contextos de intervención $(10,5 \%)$.

\subsection{Corresponsabilidad}

La participación de más de una institución en la realización de los programas es un hecho muy frecuente en Castilla y León, sobre todo a partir de 1.991, ya que en un $78,1 \%$ de los programas analizados participaba más de una entidad en su desarrollo (Tabla 2). 
TABLA 2: DISTRIBUCIÓN POR AÑOS DE LA CORRESPONSABILIDAD DE LOS PROGRAMAS PRVENTIVOS (CASTILLA Y LEÓN 1986 - 1998)

\begin{tabular}{|c|c|c|c|c|c|c|c|c|c|c|c|c|c|c||}
\hline \hline CORRESPONSABILIDAD & $\begin{array}{c}1986 \\
\%\end{array}$ & $\begin{array}{c}1987 \\
\%\end{array}$ & $\begin{array}{c}1988 \\
\%\end{array}$ & $\begin{array}{c}1989 \\
\%\end{array}$ & $\begin{array}{c}1990 \\
\%\end{array}$ & $\begin{array}{c}1991 \\
\%\end{array}$ & $\begin{array}{c}1992 \\
\%\end{array}$ & $\begin{array}{c}1993 \\
\%\end{array}$ & $\begin{array}{c}1994 \\
\%\end{array}$ & $\begin{array}{c}1995 \\
\%\end{array}$ & $\begin{array}{c}1996 \\
\%\end{array}$ & $\begin{array}{c}1997 \\
\%\end{array}$ & $\begin{array}{c}1998 \% \\
\%\end{array}$ & $\begin{array}{c}\text { TOTAL }^{(1)} \\
\%\end{array}$ \\
\hline Si & 50,0 & 28,6 & 18,2 & 57,1 & 33,3 & 90,9 & 77,8 & 78,6 & 58,3 & 81,2 & 85,7 & 84,6 & 100,0 & 78,1 \\
No & 50,0 & 0,0 & 9,1 & 42,9 & 33,3 & 9,1 & 22,2 & 21,4 & 41,7 & 18,8 & 14,3 & 15,4 & 0,0 & 21,9 \\
Desconocida & 0,0 & 71,4 & 72,7 & 0,0 & 33,3 & 0,0 & 0,0 & 0,0 & 0,0 & 0,0 & 0,0 & 0,0 & 0,0 & \\
\hline
\end{tabular}

(1) Porcentaje calculado sobre el total de programas en que se conoce esta variable.

3.3. Duración de los programas y continuidad en la implicación institucional

La duración de los programas seleccionados se distribuye de forma bastante equilibrada entre las tres categorías establecidas. Un $37 \%$ de las intervenciones preventivas tiene una duración inferior a un año, un $32,4 \%$ de uno a tres años y un $30,6 \%$ de más de tres años.

Otro dato a tener en cuenta es que de las 118 entidades que han realizado programas de prevención durante los 13 años objeto de análisis, un 34,7\% continúa desarrollándolos en la actualidad. Por otra parte, de las entidades que han intervenido en prevención a lo largo de más de un año (78 en total), un $62,8 \%$ lo hace o lo ha hecho de forma continuada.

\subsection{Objetivos y enfoque de los programas}

Tan sólo un 2,1\% de los programas analizados tenía por finalidad la reducción del daño, mientras que el 97,9\% restante tenía como objetivos prevenir el consumo de drogas.

Entre los programas de prevención del consumo de drogas el enfoque predominante es el inespecífico, que ha sido adoptado por un $40,9 \%$ de las intervenciones seleccionadas. A continuación, el enfo-

\section{GRÁFICO 3: DISTRIBUCIÓN POR AÑOS DEL ENFOQUE DE LOS PROGRAMAS DE PREVENCIÓN (CASTILLA Y LEÓN 1986 - 1998)}

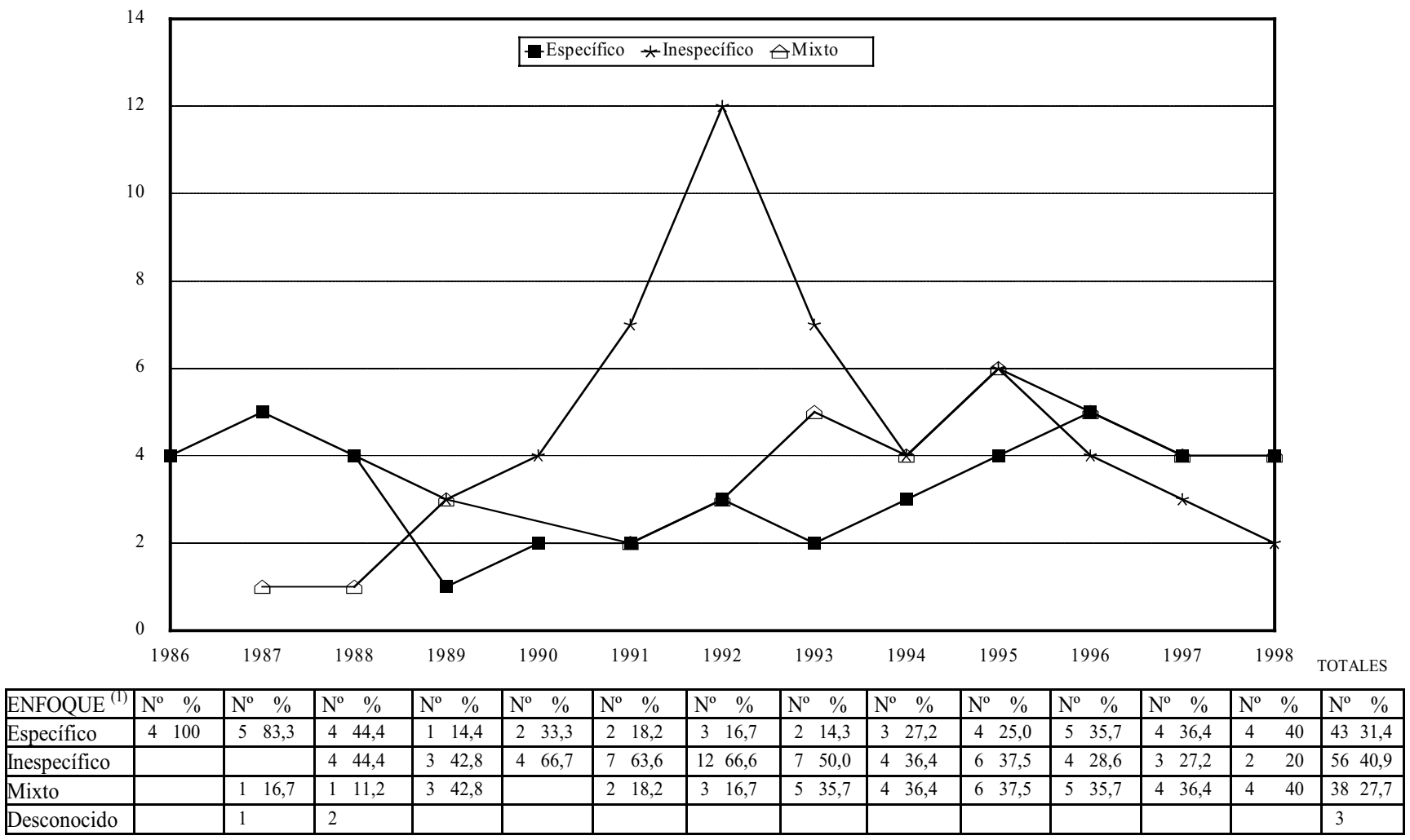

(1) Porcentaje calculado sobre el total de programas en que se conoce esta variable. 
que más utilizado ha sido el de la prevención específica, con un 31,4\% de los programas y, en tercer lugar, un enfoque mixto de prevención específica - inespecífica, que ha sido seguido por un $27,7 \%$ de las actuaciones preventivas. Al analizar la evolución temporal de los enfoques pueden distinguirse cuatro períodos: un primer período, entre 1986 y 1987, en el que predomina la prevención específica; un segundo período de transición, que cubre los años 1988 y 1989, en el que la prevención inespecífica empieza a cobrar fuerza; un tercer período, que se extiende entre 1990 y 1993, de claro predominio de la prevención inespecífica; y un cuarto y último período, a partir de 1994, en el que se observa un equilibrio en la utilización de los diferentes enfoques de prevención (Gráfico 3). que los modelos más utilizados son, por orden decreciente: promoción de alternativas (62\%), educación (54\%), información (46\%), sensibilización $(20,4 \%)$ y control de la oferta $(8,8 \%)$.

\subsection{Modelo teórico de referencia}

Uno de los aspectos más llamativos del presente análisis es que únicamente en 4 de los 143 programas analizados $(2,8 \%)$ se ha podido identificar un modelo teórico de referencia que oriente las actuaciones y que sirva para ligar de forma coherente los objetivos y las actividades del programa.

\section{TABLA 3. DISTRIBUCIÓN POR AÑOS DE LAS ESTRATEGIAS DE PREVENCIÓN UTILIZADAS POR LOS PROGRAMAS DE PREVENCIÓN DE CASTILLA Y LEÓN (1986 - 1998)}

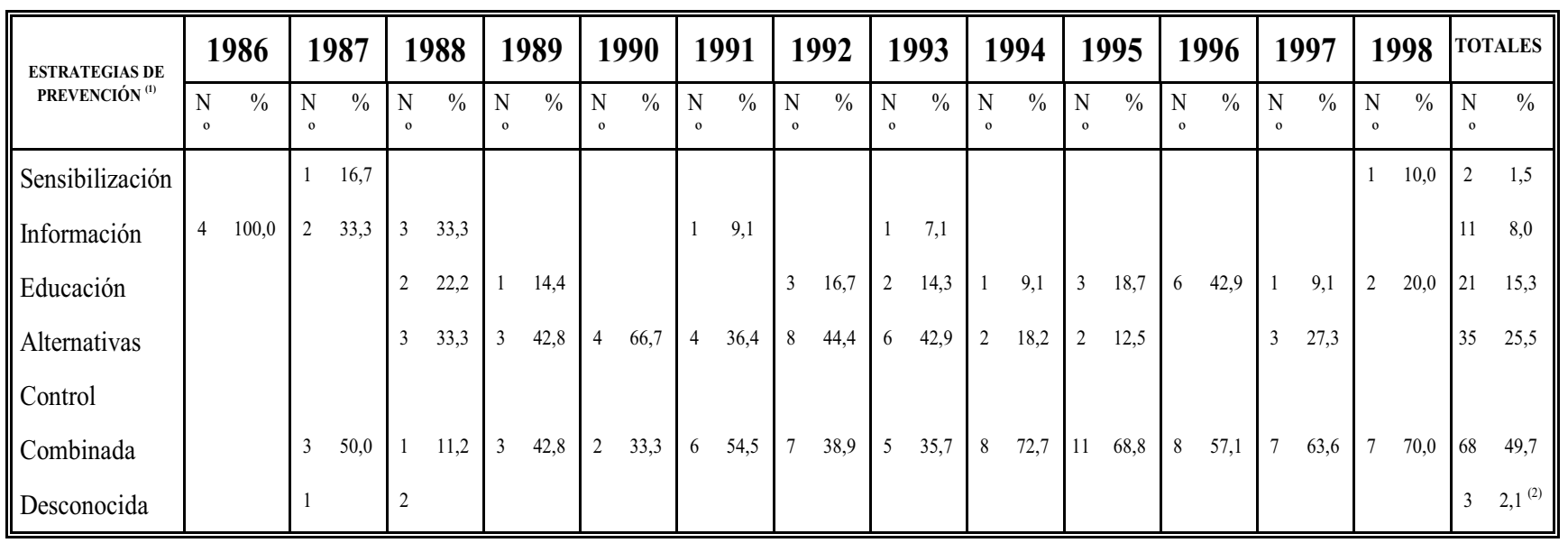

(1) Porcentaje calculado sobre el total de programas en que se conoce esta variable.

(2) Porcentaje calculado sobre el total de programas de prevención del consumo de drogas analizados.

\subsection{Estrategias de prevención}

Un 49,7\% de los programas preventivos estudiados utiliza estrategias múltiples de prevención. Dentro de esta categoría las combinaciones más frecuentes son: educación-promoción de alternativas e información-educación, representando el 12,4\% y el 6,6\%, respectivamente, del total de los programas seleccionados. Cuando las intervenciones utilizan en exclusiva una estrategia preventiva, el modelo más utilizado es el de la promoción de alternativas (25,5\%), seguido del educativo $(15,3 \%)$ y del informativo $(8,0 \%)$. Los programas preventivos basados únicamente en la sensibilización son muy minoritarios y representan tan sólo el 1,5\% de las intervenciones analizadas. Por último, no se ha identificado ningún programa basado exclusivamente en estrategias de control (Tabla 3).

Al considerar conjuntamente los programas que utilizan una combinación de estrategias y aquellos que se basan en exclusiva en alguna de ellas, se observa

\subsection{Drogas cuyos consumos se tratan de prevenir}

En un $63,7 \%$ de los casos los programas se orientan a la prevención del consumo de drogas en general, sin hacer mención de ninguna categoría de drogas o sustancia psicoactiva en particular. Un 25,8\% de las intervenciones tratan de prevenir consumos de más de una sustancia que se especifican de alguna forma, por ejemplo: alcohol y tabaco, alcohol y otras drogas, drogas legales, etc.; mientras que un 10,5\% de los programas estudiados se dirigen monográficamente a prevenir el consumo de alcohol. Precisamente, el consumo de esta sustancia es objeto de prevención en un $35,5 \%$ de los programas, bien porque las intervenciones se destinan monográficamente a esta droga, o bien porque en los programas que tratan de prevenir el consumo de más de una droga se hace mención expresa al alcohol. Aplicado este mismo criterio al tabaco, un $19,4 \%$ de los programas tienen por objeto prevenir su consumo (Tabla 4). 
TABLA 4: DROGAS CUYOS CONSUMOS TRATAN DE PREVENIR LOS PROGRAMAS PREVENTIVOS (CASTILLA Y LEÓN 1986 - 1998)

\begin{tabular}{||l|c|c||}
\hline \multicolumn{1}{|c|}{ DROGAS } & $\begin{array}{c}\text { NÚMERO DE } \\
\text { PROGRAMAS }\end{array}$ & PORCENTAJE (1) \\
\hline Drogas en general & 79 & 63,7 \\
\hline Alcohol & 13 & 10,5 \\
\hline Otras combinaciones & 32 & 25,8 \\
- Alcohol+tabaco+otras drogas & 16 & 12,9 \\
- Alcohol+ otras drogas & 7 & 5,7 \\
- Alcohol+tabaco & 5 & 4,0 \\
- Alcohol+tabaco+cánnabis & 2 & 1,6 \\
- Alcohol+tabaco+drogas de síntesis & 1 & 0,8 \\
- Drogas legales & 1 & 0,8 \\
\hline Desconocidas & 16 & $11,4^{(2)}$ \\
\hline
\end{tabular}

(1) Porcentaje calculado sobre el total de programas en que se conoce esta variable.

(2) Porcentaje calculado sobre el total de programas de prevención del consumo de drogas analizados.

\subsection{Hábitat y ámbitos de intervención}

En lo referente al hábitat, hay un claro predominio de los programas urbanos $(62,9 \%)$ sobre los de carácter rural $(26,6 \%)$. El resto $(10,5 \%)$, son programas que intervienen indistintamente en el medio urbano y rural.

Al analizar los ámbitos en los que se producen las intervenciones, se aprecia que una gran mayoría tienen la comunidad como espacio de prevención $(46,1 \%)$. No obstante, la comunidad es un ámbito heterogéneo, que en algunos casos presenta contornos indefinidos que ponen de manifiesto la falta de precisión en la definición de esta variable. Por otra parte, cuando la comunidad es un espacio bien delimi- tado engloba frecuentemente a otros ámbitos de intervención como, por ejemplo, la escuela, la familia, las asociaciones, los servicios sanitarios, los centros de trabajo, etc. El segundo contexto en el que más frecuentemente se interviene es el asociativo (asociaciones juveniles, de vecinos, de padres de alumnos, de mujeres, etc), en el que actúan un $28,1 \%$ de los programas seleccionados, le siguen: el escolar $(22,7 \%)$ y el familiar $(15,6 \%)$. Analizada la información por años, se observa que entre 1.986 y 1.990 prácticamente todos los programas giran en torno a los ámbitos comunitario, asociativo y escolar, mientras que a partir de 1.992 los contextos de intervención se diversifican mucho más, dando cabida a los medios de comunicación social, la comunidad gitana, los espacios de tiempo libre y los centros de trabajo (Tabla 5). TABLA 5: DISTRIBUCIÓN POR AÑOS DE LOS ÁMBITOS DE INTERVENCIÓN DE LOS
PROGRAMAS PREVENTIVOS (CASTILLAY LEÓN 1986 - 1998)

\begin{tabular}{|c|c|c|c|c|c|c|c|c|c|c|c|c|c|c|c|}
\hline \multirow{2}{*}{ ÁMBITOS } & 1986 & 1987 & 1988 & 1989 & 1990 & 1991 & 1992 & 1993 & 1994 & 1995 & 1996 & 1997 & 1998 & \multicolumn{2}{|c|}{ TOTAL $^{(1)}$} \\
\hline & $\mathbf{N}^{0}$ & $\mathbf{N}^{0}$ & $\mathbf{N}^{0}$ & $\mathbf{N}^{0}$ & $\mathbf{N}^{0}$ & $\mathbf{N}^{\mathbf{0}}$ & $\mathbf{N}^{0}$ & $\mathbf{N}^{0}$ & $\mathbf{N}^{\mathbf{0}}$ & $\mathbf{N}^{0}$ & $\mathbf{N}^{0}$ & $\mathbf{N}^{\circ}$ & $\mathbf{N}^{0}$ & & $\%$ \\
\hline Comunidad & 2 & 6 & 4 & 3 & 3 & 2 & 3 & 4 & 6 & 8 & 8 & 5 & 5 & & 46,1 \\
\hline Asociativo & 3 & & 2 & 2 & & 8 & 8 & 1 & 2 & 3 & 1 & 2 & 4 & 36 & 28,1 \\
\hline Escolar & 2 & & 2 & & & 1 & 3 & 2 & 3 & 4 & 2 & 5 & 5 & 29 & 22,7 \\
\hline Familiar & & & 1 & & & 2 & 3 & & 1 & 3 & 4 & 2 & 4 & 20 & 15,6 \\
\hline Espacios t. libre & & & & & & 2 & 1 & 3 & & 1 & 1 & 1 & 4 & 13 & 10,2 \\
\hline C. gitana & & 1 & & & & & 2 & & 1 & 2 & 1 & 1 & 1 & 9 & 7,0 \\
\hline MCS & & & & & & & 1 & & 1 & & 2 & 2 & 2 & 8 & 6,3 \\
\hline Laboral & & & & & 1 & 1 & & & 1 & 1 & 1 & & & & 3,9 \\
\hline Otros & & & 1 & 1 & 1 & & 7 & 2 & & 3 & 1 & 6 & 10 & & 25,0 \\
\hline Desconocido & & & 1 & 2 & 1 & & 2 & 4 & 2 & 2 & 1 & & & 15 & $10,5^{(3)}$ \\
\hline
\end{tabular}

(1) Porcentaje calculado sobre el total de programas en que se conoce esta variable.

(3) Porcentaje calculado sobre el total de programas seleccionados. 


\subsection{Destinatarios finales y mediadores de los pro-} gramas.

Los adolescentes $(66,9 \%)$, los jóvenes $(41,7 \%)$ y la población infantil $(34,5 \%)$ son los destinatarios directos o primarios a los que con más frecuencia se dirigen los programas preventivos, les siguen: la población general $(21,6 \%)$, el colectivo gitano $(7,9 \%)$ y la población adulta (8,6\%). Solamente un $4,3 \%$ de los programas se dirigen a los trabajadores, mientras que ninguna de las intervenciones analizadas se ha dirigido a la tercera edad o a la infancia temprana (0-5 años) (Gráfico 4).

\section{GRÁFICO 4: DESTINATARIOS PRIMARIOS O DIRECTOS DE LOS PROGRAMAS PREVENTIVOS (CASTILLA Y LEÓN 1986 - 1998)}

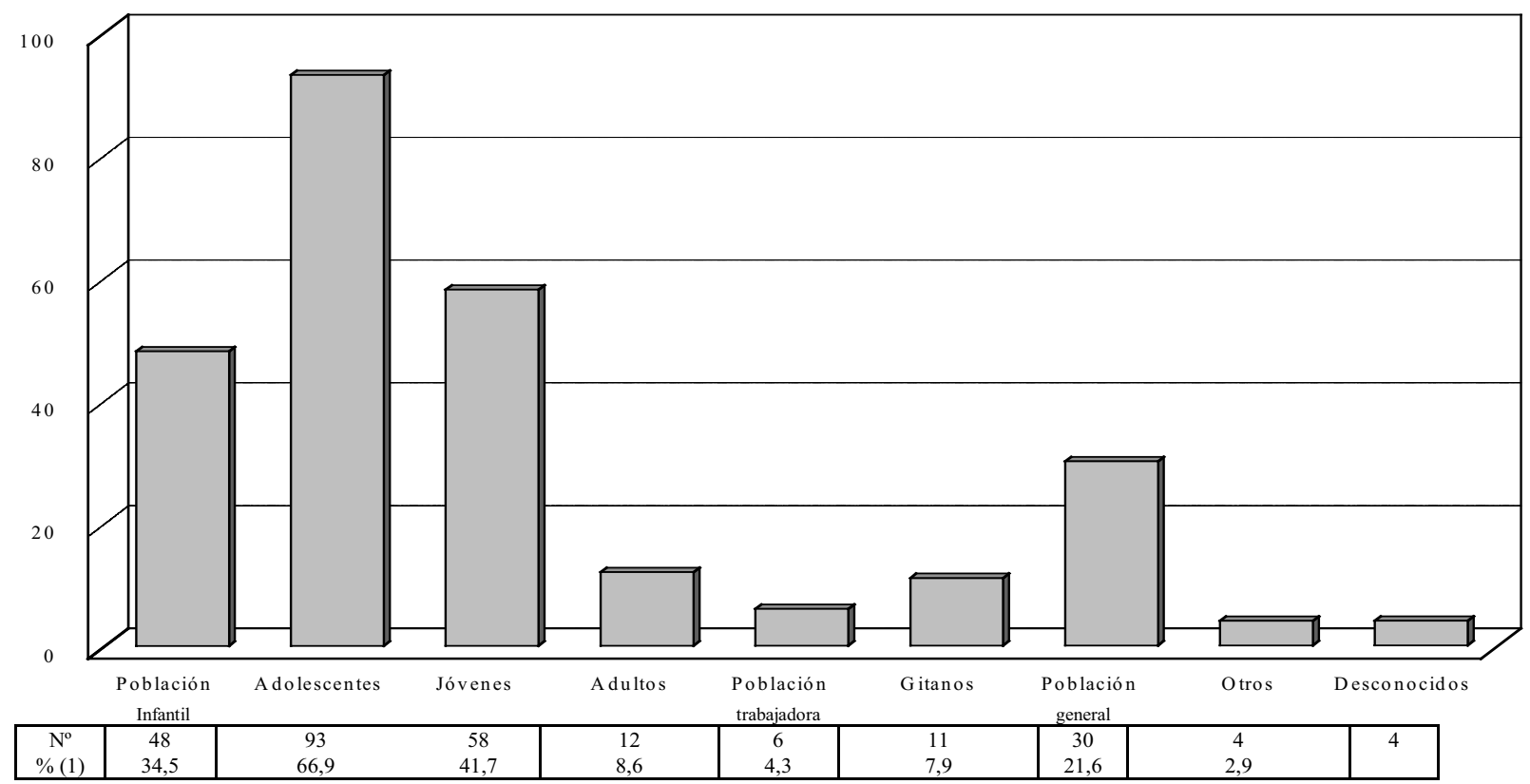

(1) Porcentaje calculado sobre el total de programas en que se conoce esta variable.

Por otro lado, un escaso $10,8 \%$ de los programas determina de forma precisa la cobertura de la población diana a la que se dirigen sus acciones preventivas y tan sólo un 9,8\% especifica sus niveles de consumo de drogas.

Otro dato a tener en cuenta es que 55 de los 140 programas seleccionados $(39,3 \%)$, se dirigen a grupos en situación de riesgo, de los cuales un 47,3\% (26 programas) define operativamente las circunstancias que concurren en la población diana para ser considerada de riesgo.
Los grupos más frecuentemente utilizados como mediadores para la prevención son, por orden de importancia: padres y madres $(37,8 \%)$, profesorado $(21,7 \%)$, monitores deportivos y de ocio y tiempo libre $(14,7 \%)$, responsables y miembros de asociaciones juveniles $(11,9 \%)$, líderes de la comunidad $(9,8 \%)$ y profesionales sanitarios $(9,1 \%)$. Otros colectivos de mediadores o de agentes de prevención pueden verse en la Tabla 6.

\section{TABLA 6: PRINCIPALES MEDIADORES DE LOS PROGRAMAS DE PREVENCIÓN (CASTILLA Y LEÓN 1986 - 1998)}

\begin{tabular}{|c|c|c|}
\hline TIPO DE MEDIADORES & $\begin{array}{l}\text { NUMERO DE } \\
\text { PROGRAMAS }\end{array}$ & PORCENTAJE ${ }^{(1)}$ \\
\hline Padres y madres & 54 & 37,8 \\
\hline Profesores & 31 & 21,7 \\
\hline Monitores deportivos y de ocio y tiempo libre & 21 & 14,7 \\
\hline Responsables y miembros de AA. juveniles & 17 & 11,9 \\
\hline Líderes de la comunidad & 14 & 9,8 \\
\hline Profesionales sanitarios & 13 & 9,1 \\
\hline Profesionales de los servicios sociales & 11 & 7,7 \\
\hline Delegados sindicales y otros mediadores del ámbito laboral & 8 & 5,6 \\
\hline Miembros de asociaciones y otros colectivos de la comunidad & 8 & 5,6 \\
\hline Voluntariado & 6 & 4,2 \\
\hline Profesionales de los medios de comunicación & 6 & 4,2 \\
\hline
\end{tabular}

(1) Porcentaje calculado sobre el total de programas en que se conoce esta variable. 
A partir de 1993 se observa una mayor diversificación en los colectivos que participan como mediadores. Por grupos, la participación de padres y madres crece considerablemente a partir de 1992 y la del profesorado a partir de 1995.

\subsection{Actividades}

El elenco de actividades preventivas utilizado por los programas es muy rico, sobre todo a partir de 1991. A lo largo de los 13 años objeto de análisis se han identificado 80 acciones preventivas distintas, con un claro predominio de las actividades de promoción de alternativas, seguidas, a considerable distancia, por las actividades de formación e información.

La utilización de uno u otro tipo de actividades ha ido evolucionando con el paso del tiempo. En los años 1986 y 1987 los programas preventivos se basaban casi en exclusiva en la información. A partir de 1988 se aprecia un alto grado de equilibrio entre acciones informativas y formativas, con un claro predominio de las actividades de promoción de alternativas desde 1991 hasta 1993. En cambio, desde 1995 los programas de prevención descansan sobre cuatro pilares de peso equilibrado: sensibilización, información, formación y promoción de alternativas (Tabla 7).

\section{TABLA 7: DISTRIBUCIÓN DE LAS ACTIVIDADES POR AÑOSY MODALIDAD PREVENTIVA (CASTILLA Y LEÓN 1986 - 1998)}

\begin{tabular}{|c|c|c|c|c|c|c|c|c|}
\hline \multirow[b]{2}{*}{ AÑ̃ } & \multicolumn{8}{|c|}{$\begin{array}{l}\text { TIPO Y NÚMERO DE ACTIVIDADES PREVENTIVAS } \\
\end{array}$} \\
\hline & Sensibilización & Información & Control & Formación & $\begin{array}{l}\text { Promoción de } \\
\text { alternativas }\end{array}$ & Comunitarias & Otras & Totales \\
\hline $\begin{array}{l}1986 \\
1987\end{array}$ & 2 & $\begin{array}{c}6 \\
11\end{array}$ & & 2 & 1 & & 1 & $\begin{array}{c}6 \\
17\end{array}$ \\
\hline $\begin{array}{r}1988 \\
1989 \\
1990 \\
\end{array}$ & 3 & $\begin{array}{l}3 \\
6 \\
1 \\
\end{array}$ & & $\begin{array}{l}3 \\
7 \\
4 \\
\end{array}$ & $\begin{array}{c}6 \\
23 \\
8 \\
\end{array}$ & $\begin{array}{l}1 \\
4 \\
1 \\
\end{array}$ & $\begin{array}{l}2 \\
4 \\
4 \\
\end{array}$ & $\begin{array}{l}15 \\
47 \\
18 \\
\end{array}$ \\
\hline 1991 & 4 & 10 & & 13 & 39 & 3 & 10 & 79 \\
\hline 1992 & 3 & 18 & 1 & 20 & 37 & 2 & 8 & 89 \\
\hline 1993 & 7 & 10 & & 12 & 33 & & 8 & 70 \\
\hline 1994 & 7 & 14 & & 10 & 21 & 3 & 7 & 62 \\
\hline 1995 & 14 & 11 & 5 & 16 & 18 & 4 & 8 & 76 \\
\hline 1996 & 6 & 12 & 1 & 13 & 18 & 9 & 10 & 69 \\
\hline 1997 & 8 & 18 & 10 & 23 & 19 & 2 & 9 & 89 \\
\hline 1998 & 6 & 19 & 5 & 19 & 25 & 8 & 8 & 90 \\
\hline
\end{tabular}

Dentro del apartado de sensibilización, las actividades más utilizadas han sido: la organización de eventos lúdicos o culturales, a las que han recurrido un $15,9 \%$ de los programas, y la celebración de días o semanas temáticas $(8,7 \%)$.

A nivel informativo, los programas se han decantado por las charlas, conferencias, jornadas o mesas redondas $(31,2 \%)$, la elaboración y/o distribución de material impreso $(27,5 \%)$, la edición de boletines, revistas o similares $(13,0 \%)$ y, en los últimos años, la realización de programas de radio y/o televisión $(8,7 \%)$.

En el terreno de la formación, los cursos y los seminarios $(49,3 \%)$ han prevalecido claramente sobre los demás tipos de actividades que forman parte de esta modalidad preventiva, siendo en términos absolutos la segunda actividad elegida. A gran distancia se sitúan las escuelas de padres y madres/escuelas de familias $(14,5 \%)$, las acciones de educación para la salud $(10,9 \%)$ y el entrenamiento en habilidades $(9,4 \%)$.

En la categoría de actividades de promoción de alternativas los talleres han sido el tipo de acción en el que se han basado la mayor parte de los programas $(50,0 \%)$. El deporte $(25,4 \%)$, la realización de excursiones y acampadas $(20,3 \%)$, la promoción de bebidas sanas $(9,4 \%)$, el teatro $(8,7 \%)$ y la incorporación de los beneficiarios directos de los programas a recursos y proyectos de la comunidad $(8,7 \%)$, han sido, a su vez, actividades frecuentemente desarrolladas en prevención.

Otro tipo de acciones preventivas relevantes han sido, por orden de importancia: la detección y captación de colectivos en riesgo (16,7\%), la constitución 
de comités de coordinación y la realización de actividades con este fin (12,3\%), juegos de diverso tipo $(10,9 \%)$, estudios diagnósticos $(10,1 \%)$ y el segui- miento y apoyo individual de los destinatarios de los programas $(9,4 \%)$ (Tabla 8$)$.

\section{TABLA 8: PRINCIPALES ACTIVIDADES DESARROLLADAS POR LOS PROGRAMAS DE PREVENCIÓN (CASTILLA Y LEÓN 1986 - 1998)}

\begin{tabular}{|c|c|c|}
\hline ACTIVIDADES & $\begin{array}{l}\text { NUMERO DE } \\
\text { PROGRAMAS }\end{array}$ & PORCENTAJE ${ }^{(1)}$ \\
\hline Talleres & 69 & 50,0 \\
\hline Cursos y seminarios & 68 & 49,3 \\
\hline Charlas, conferencias, jornadas y mesas redondas & 43 & 31,2 \\
\hline Elaboración y distribución de material impreso & 38 & 27,5 \\
\hline Deporte & 35 & 25,4 \\
\hline Excursiones y acampadas & 28 & 20,3 \\
\hline Detección y captación de colectivos en riesgo & 23 & 16,7 \\
\hline Eventos lúdicos o culturales & 22 & 15,9 \\
\hline Escuelas de padres y madres / Escuelas de familias & 20 & 14,5 \\
\hline Edición de boletines, revistas o similares & 18 & 13,0 \\
\hline Comités y actividades de coordinación & 17 & 12,3 \\
\hline Juegos de diverso tipo & 15 & 10,9 \\
\hline Educación para la salud & 15 & 10,9 \\
\hline Estudios diagnósticos & 14 & 10,1 \\
\hline Entrenamiento en habilidades & 13 & 9,4 \\
\hline Promoción de bebidas sanas & 13 & 9,4 \\
\hline Seguimiento y apoyo individual & 13 & 9,4 \\
\hline Incorporación a recursos, proyectos y actividades comunitarias & 12 & 8,7 \\
\hline Día o semana temática & 12 & 8,7 \\
\hline Teatro & 12 & 8,7 \\
\hline Programas de radio y/o TV & 12 & 8,7 \\
\hline Desconocida & 5 & $3,5^{(2)}$ \\
\hline
\end{tabular}

(1) Porcentaje calculado sobre el total de programas en que se conoce esta variable.

(2) Porcentaje calculado sobre el total de programas de prevención del consumo de drogas analizados.

\subsection{Aspectos relacionados con la evaluación}

Un 93,5\% de los programas analizados ha realizado o tiene previsto realizar algún tipo de evaluación, lo cual no es indicativo de que este aspecto de las intervenciones preventivas esté adecuadamente cubierto en Castilla y León. Así por ejemplo, de los programas que evalúan, un excepcional 1,9\% define operativamente sus objetivos, con lo que difícilmente pueden medir sus resultados si no determinan la cuantía de los cambios que quieren conseguir y el plazo de tiempo en que se quieren alcanzar.

Centrando la atención en otros aspectos de la evaluación, se observa que un $62,5 \%$ de las intervenciones define algún tipo de indicadores y un 41,4\% determina los instrumentos o métodos de evaluación, si bien es cierto que a menudo ambos elementos se establecen con poca precisión. Entre los instrumentos y métodos de evaluación, los más frecuentemente utilizados son: la observación, las entrevistas y las reuniones, las hojas de registro y de seguimiento individual, las escalas y los cuestionarios. 
Por último, un 40,9\% de los programas define sus fuentes de información, un 26,4\% concreta un calen- dario de evaluación y un ínfimo 1,9\% utiliza algún diseño de investigación para la evaluación (Tabla 9).

\section{TABLA 9: ASPECTOS RELATIVOS A LA EVALUACIÓN DE LOS PROGRAMAS DE PREVENCIÓN (CASTILLA Y LEÓN 1986 - 1998)}

\begin{tabular}{||l|c|c||}
\hline \multicolumn{1}{|c|}{ ASPECTOS DE LA EVALUACIÓN } & $\begin{array}{c}\text { NUMERO DE } \\
\text { PROGRAMAS }\end{array}$ & PORCENTAJE ${ }^{\text {(4) }}$ \\
\hline Ha realizado o tiene prevista evaluación & 116 & 93,5 \\
Definen los objetivos operativamente & 2 & 1,9 \\
Determinan indicadores de evaluación & 70 & 62,5 \\
Establecen instrumentos o métodos de evaluación & 46 & 41,4 \\
Concretan las fuentes de información & 45 & 40,9 \\
Marcan un calendario de evaluación & 28 & 26,4 \\
Utilizan un diseño de investigación & 2 & 1,9 \\
\hline
\end{tabular}

(4) Porcentaje calculado sobre el total de programas que evalúam y en los que se ha podido obtener información de esta variable.

A diferencia de lo que se ha observado con otros aspectos de los programas de prevención la evaluación no ha experimentado cambios consistentes en los 13 años en los que se ha centrado el estudio.

\section{DISCUSIÓN}

En España y en Castilla y León se han producido una serie de hechos positivos para la prevención que constituyen una buena base sobre la que ir refinando progresivamente los programas en los próximos años. Varios argumentos apoyan esta afirmación.

El primero de ellos es que durante los últimos años se ha alcanzado una considerable implicación de la iniciativa pública y privada en prevención (GID, 1997). En Castilla y León, la Administración Autonómica ha desempeñado básicamente el papel de entidad promotora y financiadora de los programas, mientras que los Ayuntamientos (sobre todo los de más de 20.000 habitantes) han sido la principal institución ejecutora de los mismos.

Por otro lado, cada vez son mas las entidades e instituciones que entienden que la prevención es una tarea compartida entre distintas instancias de la sociedad, como lo pone claramente de manifiesto el alto grado de corresponsabilidad detectado en el desarrollo de los programas.

Dos circunstancias favorables que se añaden a las anteriores y que se han detectado igualmente a nivel nacional (GID, 1997) son, por un lado, que el compromiso de las instituciones en prevención suele estar presidido por una relativa estabilidad y continuidad en el tiempo y, por otro lado, que el volumen de actividad preventiva ha experimentado un crecimiento más que notable en las últimas décadas. En Castilla y León esta expansión preventiva se detecta sobre todo a partir de 1992.

Otro elemento positivo que merece ser destacado es el énfasis que han puesto los programas de prevención en las drogas institucionalizadas, en especial en la prevención del consumo de alcohol.

Los programas de prevención en Castilla y León han experimentado una profunda transformación durante estos 13 años, hasta el punto de que es posible distinguir tres etapas o momentos diferenciados.

La primera etapa se extiende entre 1986 y 1988. En esta etapa las actuaciones de prevención fueron desarrolladas mayoritariamente por ONGs. específicas del campo de las drogodependencias, consistiendo básicamente en charlas, conferencias y actividades informativas específicas de similares características. Por lo general, las actividades preventivas tenían la comunidad como contexto de intervención y se dirigían a todo el conjunto de la población, considerándose ambos aspectos como un todo indiferenciado sin definición ni límites precisos.

La segunda etapa transcurre tras dos años de transición y cubre el período comprendido entre 1991 y 1994, coincidiendo en gran medida con la vigencia del II Plan Regional sobre Drogas. En esta fase se produ- 
ce un crecimiento muy acusado de la actividad preventiva, gracias, sobre todo, a la importante implicación de los Ayuntamientos de la Comunidad Autónoma. Son características propias de este período que una gran parte de los programas se desarrollan con la participación de más de una entidad y que hay un claro predominio de la prevención inespecífica a través de la promoción de alternativas al consumo de drogas. Por otra parte, se aprecia una diversificación de los ámbitos de intervención y un enriquecimiento de las actividades preventivas que se desarrollan en el marco de los programas.

La tercera, y última etapa, se inicia en 1994, a raíz de la aprobación de la Ley 3/1994, y perdura hasta el momento actual. Durante esta fase se mantiene el nivel de actividad preventiva y la implicación municipal, desarrollándose los programas con un alto grado de corresponsabilidad entre las entidades implicadas en prevención. Las interveciones se caracterizan por un equilibrio en la utilización de los diferentes enfoques preventivos existentes, hasta el punto de que más de una tercera parte opta por un encuadre mixto de prevención específica e inespecífica. La combinación de estrategias preventivas y la diversificación de los mediadores que participan en los programas también son elementos característicos de este período. En lo referente a las actividades, se empiezan a incorporar las medidas de control y los programas combinan de una forma equilibrada acciones diversas de sensibilización, información, educación y promoción de alternativas, sin que predomine claramente ningún tipo de actividades sobre las demás.

En resumen, la prevención hoy, tanto en Castilla y León como en España, se caracteriza por un gran volumen de actividad basada en múltiples estrategias (muchas veces combinadas), que van más allá de la mera sensibilización e información. Igualmente, se puede afirmar que de esta actividad preventiva se ocupa un número creciente de entidades e instituciones y que en ella participan profesionales y voluntarios de la comunidad que han recibido formación específica para cumplir con este cometido (GID, 1997).

De las consideraciones anteriores no se debe extraer una conclusión triunfalista ni la idea de que la prevención en España y en Castilla y León ha resuelto la mayor parte de sus problemas. Muy por el contrario, del presente estudio y de otros de similares características realizados a nivel nacional, se desprende que las intervenciones preventivas presentan numerosos puntos débiles y deficiencias que se deben ir corrigiendo sin dilación en los próximos años (Zacagnini, Colom y Santacreu, 1.993; Froján y Santacreu, 1.994; March Cerdá y Orte, 1.994; y GID, 1997).

Una de las principales insuficiencias detectadas en los programas de prevención en España es la ausencia de modelos teóricos que orienten las intervenciones y den coherencia a los objetivos, estrategias, métodos y actividades del programa (Froján y Santacreu, 1994; March Cerdá y Orte, 1994). De hecho, se observa un cierto mimetismo en los programas, un cierto grado de estandarización, que no responde a las necesidades, problemas y obstáculos detectados en cada zona concreta de intervención ni a las líneas de intervención preventiva que se desprenden de los modelos teóricos con mayor aval empírico.

La formulación de objetivos es una de las deficiencias detectadas en el plano metodológico de los programas de prevención en Castilla y León, que también ha sido señalada a nivel estatal en el estudio del GID de 1997. Los objetivos no se definen en términos operativos, lo que hace muy difícil y en muchos casos imposible la evaluación de la eficacia del programa. El presente estudio ha puesto de manifiesto que muchos de los programas no determinan con precisión el tipo de consumos de drogas que tratan de prevenir ni la naturaleza de los cambios que tratan de conseguir, es decir, si sólo tratan de incidir en las conductas de consumo de drogas o también en variables mediadoras estrechamente ligadas con los hábitos de consumo. Por ejemplo, nivel de información, creencias, actitudes y valores favorables a la salud y al establecimiento de vínculos sociales positivos, toma de decisiones, habilidades de relación interpersonal, autoestima, etc.

Las carencias técnicas en el diseño de las intervenciones también afectan a la definición de la población destinataria. No es frecuente que los programas determinen su cobertura ni que definan con precisión las características del grupo en el que se quieren introducir los cambios preventivos. Por ejemplo, se omiten detalles sobre si la población diana consume o no drogas y en qué grado, o si se trata de colectivos de riesgo y por qué, en cuyo caso las acciones preventivas deberían empezar antes, ser más intensas y aplicarse durante más tiempo.

La estandarización de los programas también se aprecia al analizar los destinatarios directos de las intervenciones. La gran mayoría (y este es un dato positivo) se dirige a los grupos de edad más susceptibles de iniciarse en el uso, consumo habitual y abuso de drogas, esto es, la población adolescente y joven. La otra cara de la moneda es que muy pocos programas se preocupan de la población adulta o de la población trabajadora, por sólo citar dos ejemplo significativos.

Una de las dificultades con las que más consistentemente se enfrentan los programas de prevención, tanto en España como en la esfera internacional, es la captación y el mantenimiento de la población destinataria en los programas. Parece como si los potenciales beneficiarios de las acciones preventivas estuvieran afectados por lo que se podría llamar "ilusión de inmunidad", es decir, que a pesar de considerar que las drogodependencias son un problema social grave, 
tuvieran al mismo tiempo la percepción de que a ellos no les va a afectar o, quizá, según lo propuesto por el modelo de creencias de salud (Salleras, 1991), alberguen la convicción de que las actuales respuestas preventivas no son del todo eficaces para evitar el problema. Sea como fuere, el hecho cierto es que la gran mayoría de los programas no contempla actuaciones específicas de reclutamiento y de motivación de los destinatarios para mantenerse bajo la influencia de las acciones de prevención.

Otra importante limitación, que afortunadamente parece haber empezado a solventarse en Castilla y León durante la segunda mitad de la década de los 90, es la falta de planificación de las actuaciones preventivas. Por sólo citar un ejemplo, la gran mayoría de las intervenciones carece de estudios diagnósticos previos, con lo que es perfectamente posible que se desarrollen programas de prevención primaria con poblaciones que consumen regularmente drogas, o que se apliquen programas de prevención universal (NIDA, 1.997) para poblaciones que se encuentran en situación de riesgo.

La falta de fundamentación teórica y de planificación de las actuaciones preventivas, que es detectable tanto en los programas que se realizan en Castilla y León como en España (March Cerdá y Orte, 1994; GID, 1997), son carencias importantes que convierten la acción preventiva en una actividad "a ciegas", o dicho en otros términos, en una metodología intuitiva en la que con la simple intervención, como un disparo en la oscuridad que intenta dar en el blanco, se espera cambiar, reducir o eliminar los comportamientos relacionados con el consumo de drogas (Cázares, 1997).

A pesar de que las instituciones implicadas en prevención en Castilla y León presentan un estimable grado de estabilidad y continuidad en sus programas y de que casi un $31 \%$ de las intervenciones tiene una duración de más de tres años, las actuaciones puntuales o escasamente sistemáticas suelen ser frecuentes. El análisis ha puesto de manifiesto que muchos programas tienen un carácter cíclico, es decir, se desarrollan en una determinada época del año (por ejemplo, el verano); son programas que intervienen en períodos muy concretos y, en ocasiones, en espacios muy cortos de tiempo. Por otro lado, también se ha podido constatar que, con cierta frecuencia, las instituciones cambian de estrategia o programa preventivo sin que haya razones muy claras para ello, interrumpiendo así líneas de acción preventiva que ya estaban en marcha.

Otra laguna que se ha puesto de manifiesto en el presente análisis es la insistencia en algunas acciones de dudosa eficacia preventiva, mientras que otras con un considerable aval empírico se marginan sistemáticamente de los programas. En el haber de las primeras tan sólo se encuentra la facilidad de su aplicación y la amplia aceptación social e institucional con que cuentan, razones ambas que parecen tener el suficiente peso para que se demanden y repitan una y otra vez.

Si bien es cierto que en los últimos años se ha observado una evolución positiva en España (Froján y Santacreu, 1994; GID, 1997), aún se aprecia una considerable influencia del discurso voluntarista de "todo vale" en prevención. Este discurso se constata en la frecuente utilización de ciertas actividades alternativas de carácter inespecífico, cuya relación con los factores de riesgo y de protección del consumo de drogas y su virtualidad preventiva no ha sido establecida. En la proliferación de estas actividades en Castilla y León, especialmente intensa entre 1988 y 1993, no ha sido ajeno el hecho de intentar obtener financiación extraordinaria para otros programas con cargo a los presupuestos del Plan Regional sobre Drogas.

Por otra parte, las actividades de formación, que con tanta frecuencia se han utilizado en los programas de prevención de la Comunidad Autónoma, se han dirigido mayoritariamente a la capacitación de mediadores para la prevención. Sin embargo, los profesionales y voluntarios de la comunidad formados para intermediar no han llegado en muchos casos a desempeñar el rol de agentes de prevención con los destinatarios primarios de los programas. En la práctica, una supuesta actividad preventiva se ha quedado en una simple acción de formación. En este mismo sentido, un dato revelador del desequilibrio existente en las estrategias de formación es la escasa presencia de actividades de entrenamiento en habilidades en los programas preventivos.

Diversos estudios han puesto de manifiesto que el desinterés por la evaluación es un mal común que afecta de modo generalizado a la prevención en España (Froján y Santacreu, 1994; March Cerdá y Orte, 1994 y GID, 1997). Por lo general, la evaluación de los programas preventivos se plantea de una forma muy limitada y con escasa precisión y rigor. En la mayor parte de las intervenciones se omite la evaluación de los resultados, y cuando se hace evaluación, ésta se centra casi siempre en los procesos, olvidándose, aún en este caso, de aspectos importantes que tienen que ver con el control de la implementación y ejecución de los programas. Por el contrario, es frecuente que las evaluaciones se centren en el grado de actividad del programa y en la satisfacción de los beneficiarios con las acciones preventivas.

Otra de las lagunas mas generalizadas de la evaluación es la ausencia de diseños de investigación, de modo que los cambios observados no pueden atribuirse con la necesaria credibilidad científica a la aplicación del programa. Esta insuficiencia también tiene su importancia, pues hace que no sea posible avanzar en el conocimiento de los enfoques y planteamientos que resultan más eficaces para reducir la demanda de 
drogas y que, por lo tanto, deben adoptarse y perfeccionarse en el futuro.

Comparando los resultados del presente estudio con otros análisis descriptivos realizados a nivel nacional, cabe concluir que las actuaciones preventivas desplegadas en Castilla y León en los últimos 13 años participan de similares deficiencias metodológicas que otros programas de prevención aplicados en el Estado español; deficiencias que afectan especialmente a la planificación y a la evaluación de las intervenciones (Zacagnini, Colom y Santacreu, 1.993; Froján y Santacreu, 1.994; March Cerdá y Orte, 1.994; y GID, 1997).

De todo lo dicho con anterioridad se desprende que es necesario avanzar hacia una segunda generación de programas de prevención en la que se depuren las deficiencias antes enumeradas. Se dispone en la actualidad de una buena base sobre la que trabajar en la reducción de la demanda y, como el escultor que poco a poco va moldeando y puliendo la materia prima hasta concluir su obra, es preciso avanzar en una línea de perfeccionamiento que permita entrar en el siglo XXI con unos programas más eficaces de prevención basados en la investigación básica y aplicada.

\section{BIBLIOGRAFÍA}

Cázares, A. (1997). Investigación sobre intervenciones preventivas: enfoque y perspectiva. En Métodos científicos para la investigación de intervenciones preventivas. Madrid. Fundación de Ayuda contra la Drogadicción (FAD).

Froján, M. J. y Santacreu, J. (1994). Evaluación de programas de prevención del consumo de drogas. Adicciones. Vol. 6, n 3: 283-299.

Grupo interdisciplinar sobre drogas (GID). (1997). Revisión histórica de los programas de prevención de las drogodependencias en España. En Prevención de las drogodependencias. Análisis y propuestas de actuación. Ministerio del Interior. Delegación del Gobierno para el Plan Nacional sobre Drogas (DGPNSD). Madrid.
Idea Prevención. (1998). Cuestionario de participación en el Sistema de información técnica sobre prevención del abuso de drogas. Boletín de Idea Prevención n 17.

Junta de Castilla y León. Consejería de Cultura y Bienestar Social (1989). Plan Regional sobre Drogas. Valladolid.

Junta de Castilla y León. Consejería de Sanidad y Bienestar Social. (1992). II Plan Sectorial sobre Drogas. Valladolid. Junta de Castilla y León. Consejería de Sanidad y Bienestar Social. (1995). Ley de Prevención, Asistencia e Integración Social de Drogodependientes de Castilla y León, Ley 3/ 1994, de 29 de marzo. Valladolid.

Junta de Castilla y León. Consejería de Sanidad y Bienestar Social. (1998). III Plan Sectorial sobre Drogas. Valladolid.

March, M. y Orte. C. (1994). Revisión de los programas de prevención de las drogodependencias en España: un análisis desde Idea-Prevención. Boletín Idea-Prevención. $n^{\circ}$ 9: 77-83.

Ministerio de Sanidad y Consumo. (1985). Plan Nacional sobre Drogas. Madrid.

Delegación del Gobierno para el Plan Nacional sobre Drogas (DGPNSD). (1997). Criterios básicos de intervención en los programas de prevención de las drogodependencias. En Prevención de las drogodependencias. Análisis y propuestas de actuación. Ministerio del Interior. Madrid.

National Institute on Drug Abuse. (1997). Preventing drug use among children and adolescents. A research-based guide. National Institute of Health (NIH). Publication NO. 97-4212.

Salleras, L. (1991). Educación Sanitaria: concepto, campos de acción, agentes y las bases científicas de la modificación de los comportamientos de salud. En Medicina preventiva y Salud Pública. Barcelona. Masson-Salvat Medicina.

Zaccagnini, J.L., Colom, R. y Santacreu, J. (1993). Catálogo de programas de prevención de la drogadicción. Valencia. Promolibro 
